\title{
Is smoking behaviour in patients with coronary heart disease influenced by whether their attending physician smokes?
}

\author{
Kunihisa Miwa, Masatoshi Fujita, Yuko Miyagi, Hiroshi Inoue, Shigetake Sasayama
}

\begin{abstract}
Objective-To examine the association between the smoking status of attending physicians and that of their patients.

Setting-Cardiology division of the Toyama Medical and Pharmaceutical University Hospital and two affiliated hospitals in Toyama District, Japan.

Subjects-A total of 259 patients with known ischaemic heart disease who consulted our outpatient clinic in September 1992, and their attending physicians at the clinic $(n=17)$. Thirty eight attending physicians who cared for 100 of these patients during a previous admission of the University Hospital were also included in the study.

Design and main outcome measures Smoking status of patients was determined by urinary cotinine radioimmunoassay using ${ }^{125}$ I-cotinine. Patients with urinary cotinine concentration above $50 \mathrm{ng} / \mathrm{ml}$ were classified as smokers. Smoking status of physicians was determined by self report.
\end{abstract}

Results-Smoking was found to be significantly more prevalent $(p<0.05)$ in outpatients whose attending physicians smoked $(20 / 94,21.3 \%)$ than in those with non-smoking attending physicians $(20 / 165,12.1 \%)$. When 100 patients who had been admitted to our hospital because of ischaemic heart disease were also classified according to their attending physicians' smoking status during their admission, smoking was found to be significantly more prevalent $(p<0.05)$ in those whose attending physicians smoked $(7 / 28,25.0 \%)$ than in those with a non-smoking physician $(7 / 72$, 9.7\%).

Conclusions-Smoking prevalence was higher among patients whose attending physician smoked than among those with a non-smoking physician. The cross sectional nature of the study does not allow a definitive conclusion about cause, but the findings suggest that physicians' smoking status affects the smoking status of their patients with ischaemic heart disease.

(Tobacco Control 1995; 4: 236-238)

Keywords: smoking among physicians; smoking among patients; coronary heart disease
Introduction

Smoking has been established as an important risk factor for ischaemic heart disease. In Japan, the prevalence of smoking in male adults is as high as $60 \%$. The smoking prevalence is also high $(30-40 \%)$ in Japanese physicians, and is not rare even among cardiologists. ${ }^{1}$ Epidemiological studies have shown that smoking cessation significantly improves the prognosis of patients with ischaemic heart disease. ${ }^{2}$ Education regarding smoking cessation is crucial to improve the outcome in such patients. Although it has been suggested that physicians should play an important role in smoking cessation education, ${ }^{3}$ enthusiasm for patient smoking cessation instruction appears to vary among physicians. ${ }^{4}$ Smoking among physicians themselves may dampen such enthusiasm. The purpose of this study was to determine whether smoking among attending physicians is associated with smoking among their patients.

\section{Methods}

The cardiology division of the Toyama Medical and Pharmaceutical University hospital and two affiliated hospitals in Toyama district (Shakaihoken Takaoka hospital and Himekawa hospital) were involved in this study. Patients with known ischaemic heart disease who consulted one of these outpatient cardiology clinics in September 1992 were automatically enrolled in this study. Patients with serious current complications or systemic disease were excluded.

The study subjects comprised a total of 259 patients including 181 men and 78 women, with a mean age of 63 years. Old myocardial infarction was seen in 93 , angina pectoris in 142 , and other ischaemic heart disease in 24 patients. The 17 attending physicians of these patients were all cardiologists $(16$ males and one female), nine in the University Hospital, four in the Shakaihoken Takaoka Hospital, and four in the Himekawa Hospital. Urine samples were collected from all the patients. Although the doctors and patients enrolled in this study were informed that the urine samples were used to determine their smoking status, the purpose of the study was not made clear to them throughout its duration.

Urine was stored at $-20^{\circ} \mathrm{C}$ until assayed. Urinary cotinine, a major and stable metabolite 


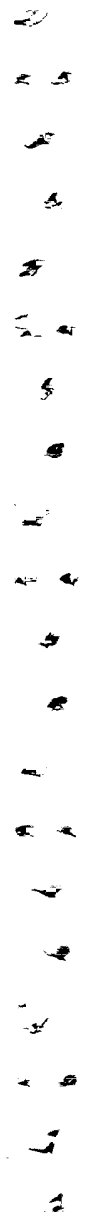

2

$\bar{x}$

$-$

$\mathbf{x}$

$*$

12

$\leq \leq$

$\checkmark$

; Smoking status determined by urinary cotinine assay among patients with ischaemic heart disease

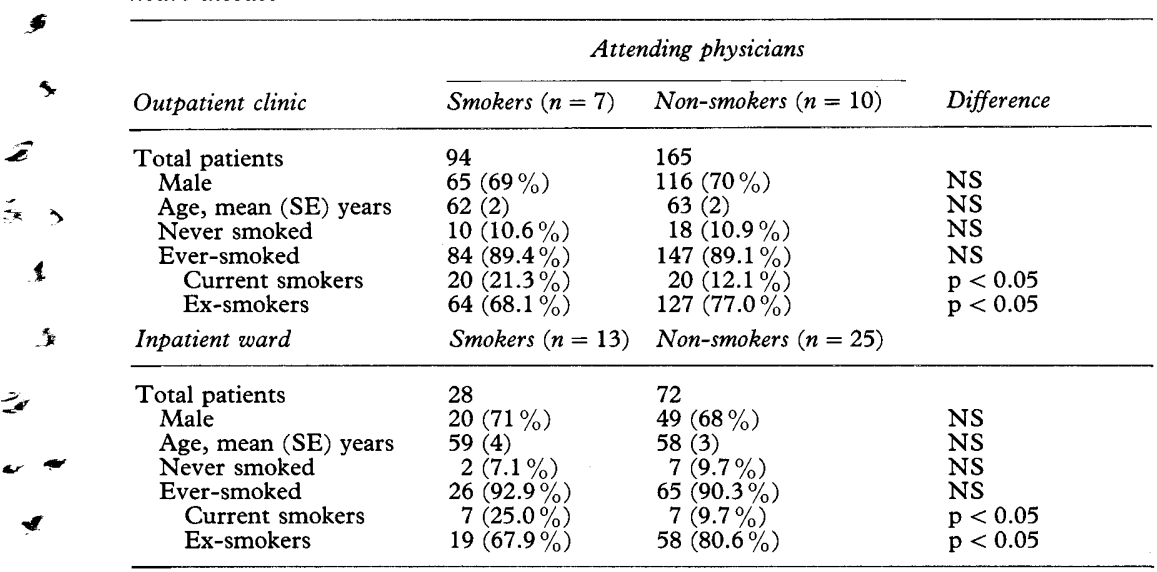

of nicotine, was measured by radioimmunoassay with the use of ${ }^{125}$ I-cotinine and anticotinine antisera from rabbits, as described previously..$^{5,6}$ Under usual conditions, the urinary cotinine concentration was consistently below $50 \mathrm{ng} / \mathrm{ml}$ in non-smokers, while in smokers who smoked three or more cigarettes a day, the concentration was always above $100 \mathrm{ng} / \mathrm{ml} .^{5}$ Therefore patients with urinary cotinine concentrations above $50 \mathrm{ng} / \mathrm{ml}$ were classified as current smokers in this study.

Current non-smokers were subdivided into patients who had never smoked and exsmokers according to self report. Patients were then divided into two groups depending on the smoking status of their attending physicians at the outpatient clinic. Of these patients, 100 had experienced previous admission to the University hospital because of ischaemic heart disease; these patients were subdivided according to the smoking status of their attending physicians during their hospital admission: 38 doctors ( 31 males and seven females) consisting of 22 cardiologists and 16 residents in internal medicine.

The smoking status of physicians was determined by self report. We asked physicians whether they routinely asked their patients if they smoked and whether they routinely gave advice on stopping smoking to smoking patients.

\section{Results}

Results are shown in the table. The outpatient clinic cardiologists included seven current smokers and 10 non-smokers (including one ex-smoker). Twenty ( $21.3 \%$ ) of the 94 patients attended by smoking physicians were identified as smokers by urinary cotinine assay. In contrast, among the 165 patients attended by non-smoking physicians, $20(12.1 \%)$ were smokers. The prevalence of smoking was significantly higher $(p<0.05)$ in patients attended by smoking cardiologists than by non-smoking cardiologists. Ex-smokers were significantly less prevalent $(68.1 \%, 64 / 94)$ among patients attended by smoking cardiologists than among patients attended by non- smoking cardiologists $(77.0 \%, 127 / 165)(\mathrm{p}<$ 0.05).

Of 100 patients who were admitted to our University hospital because of ischaemic heart disease, 28 were attended by smoking physicians, and 72 by non-smoking physicians. The prevalence of smoking among patients attended by smoking physicians was $25.0 \%$ $(7 / 28)$; this was higher $(p<0.05)$ than that among patients attended by non-smoking physicians $(9.7 \%, 7 / 72)$. Ex-smokers were less prevalent among patients attended by smoking physicians $(67.9 \%, 19 / 28)$ than among patients attended by non-smoking physicians $(80.6 \%, 58 / 72)(p<0.05)$.

Four $(40 \%)$ of the outpatient non-smoking physicians and five $(20 \%)$ of the inpatient non-smoking physicians said that they repeatedly asked patients about smoking and routinely gave advice on quitting to smoking patients. In contrast, only one physician $(14 \%)$ among the outpatient smoking physicians and two $(15 \%)$ among the inpatient smoking physicians addressed patient smoking in this way.

\section{Discussion}

Our study showed that the prevalence of smoking in patients with ischaemic heart disease differed according to the smoking status of their attending physicians. While there was no difference in the prevalence of previous or current smoking among patients according to the smoking status of their attending physicians, the percentage of exsmokers was significantly lower when the attending physicians were smokers. Although this tendency was observed in both the outpatient clinic and inpatient ward, the effect of the physician's smoking status on patient behaviour appeared to be somewhat stronger in the inpatient ward than in the outpatient clinic. These results suggest that the smoking status of the attending physician may affect patient smoking status, and therefore the prognosis of patients with ischaemic heart disease. As smoking cessation is crucial to improve the long term prognosis of patients with ischaemic heart disease, smoking cessation education by physicians is important.

The higher smoking prevalence in patients attended by smoking physicians may be due, at least in part, to less intensive smoking cessation counselling by these physicians, because physicians with a favourable attitude toward smoking cessation education were rare among the attending physicians who smoked. Aasen and Gulsvik ${ }^{7}$ reported that physicians who had stopped smoking routinely asked their patients about smoking and more often gave patients advice on drug treatment to help them stop smoking than non-smoking physicians. For physician ex-smokers, long term health risks were the most important reason for giving up smoking, followed by the desire to set an example as medical personnel.

Japan still has extremely high smoking rates in males compared with other developed countries, though much lower rates in females. 
According to a report by Japan Tobacco, about $60 \%$ of male and $14 \%$ of female adults are current smokers in Japan. The anti-smoking movement in Japan has started quite recently and has so far had little governmental support. About $44 \%$ of Japanese general practitioners are current smokers. ${ }^{4}$ In contrast, the smoking prevalence among doctors has decreased dramatically in many Western countries. For example, smoking prevalence is $4 \%$ among general practitioners in the United Kingdom and $9 \%$ among all physicians in the United States. ${ }^{4}$

Smoking prevalence has declined very slowly among Japanese doctors. Smoking prevalence among Japanese cardiologists was reported to be $68 \%$ in $1965,52 \%$ in 1979 , and $37 \%$ in $1988 .{ }^{8}$ Smoking prevalence among doctors in Japan is now more than four times higher than that in Western countries, whereas they were comparable 40 years ago. In our study we found a smoking prevalence rate of nearly $40 \%$ for physicians in our institute, the average rate among Japanese doctors. Kawane and Soejima reported that $24 \%$ of the physicians in their institute are smokers and a higher smoking prevalence among medical students and young physicians. ${ }^{9}$ Smoking prevalence among male medical students is $40-50 \%$ in Japan, while it is under $10 \%$ in the United States and Australia. ${ }^{10}$ It has been reported that high proportions of medical students in Australia and the United States, but a much lower proportion in Japan, would give routine advice on quitting to smoking patients. ${ }^{10}$

Our study had several limitations. Our cross sectional design does not allow us to establish a causal relationship between the smoking status of attending physicians and their patients. If the relationship is causal, it is unclear why the smoking status of patients was influenced by that of their attending physicians. The smoking cessation techniques used by physicians were not evaluated or compared between smoking and non-smoking attending physicians. Finally, we cannot exclude the possibility that patients who are attended by smoking physicians differ from those seen by non-smoking physicians. Various factors that may influence the likelihood of patients continuing to smoke, such as sociodemographic status, motivation to quit, comorbidity, etc, were not assessed in our study. It cannot be ruled out that smokers were turned away by non-smoking physicians, or that non-smokers were turned away by smoking physicians; however, that was not the case in the inpatient ward because in Japanese inpatient settings, patients are not permitted to change attending physicians, nor can attending physicians turn away patients.

In conclusion, smoking prevalence among patients with ischaemic heart disease is higher for those attended by physicians who smoke than for those attended by non-smoking physicians. Tobacco smoking is inappropriate for those who wear white coats. A reduction in smoking prevalence among physicians would probably promote smoking cessation among patients with ischaemic heart disease, which would in turn result in a more favourable prognosis.

This study was supported in part by grants from the Japan Research Foundation for Clinical Pharmacology, Tokyo, Japan. Anticotinine antisera obtained from rabbits were kindly provided by Professor Shigeru Matsukura of Miyazaki Medical University, Japan.

1 Kawane H. Tobacco smoking in Japan. Can Med Assoc 7 $1986 ; 135: 971$.

2 US Department of Health and Human Services. The health benefits of smoking cessation. A report of the Surgeon General, 1990. Rockville, Maryland: Public Health Service, Centers for Disease Control, Office on Smoking and Health, 1990. (DHHS Publication No (CDC) 90-8416.)

3 Jones MA, Oates JA, Ockene JK, Hennekens CH. Statement on smoking and cardiovascular disease for health care professionals. Circulation 1992; 86: 1664-9.

4 Davis RM. When doctors smoke. Tobacco Control 1993; 2: 187-8.

5 Miwa K, Miyagi Y, Asanoi H, Fujita M, Sasayama S. Augmentation of education for smoking cessation by urinary cotinine measurement. $\mathcal{f} p n$ Circ $\mathcal{f} 1993$; 57: 775-80.

6 Miwa K, Fujita M, Miyagi Y. Beneficial effects of smoking cessation on the short-term prognosis for variant angina - validation of the smoking status by urinary cotinine measurements. Int f Cardiol 1994; 44: 151-6.

7 Aasen T, Gulsvik A. Smoking and giving of patient information by chest physicians. Eur $\mathcal{F}$ Respir Dis 1986; 69: 215-8.

8 Matsuzaki M. Igakunoayumi 1993; $167: 853$ (in Japanese).

Kawane H, Soejima R. Smoking among the staff of medical school hospital. In: Aoki M, et al, eds. Smoking and health 1987. Amsterdam: Elsevier Science Publishers, 1988: 685-7.

10 Tessier J-F, Fréour P, Nejiari C, Belougne D, Crofton JW. Smoking behaviour and attitudes towards smoking of medical students in Australia, Japan, USA, Russia, and Estonia. Tobacco Control 1993; 2: 24-9. 